\title{
Convenient Creation and Use of Suturing Supplies for Laparoscopic Partial Nephrectomy
}

\author{
J. Stuart Wolf, Jr., M.D.
}

\begin{abstract}
The closure of the renal defect is the most challenging part of laparoscopic partial nephrectomy when suturing is required. The author presents a method of creating and using suturing material for laparoscopic partial nephrectomy that has been developed over several hundred cases at the author's institution. These techniques can be used to simplify the task of suturing during laparoscopic partial nephrectomy.
\end{abstract}

\section{Introduction}

L APAROSCOPIC PARTIAL NEPHRECTOMY is gradually becoming a standard of care for small renal masses. The limiting factors are tumor characteristics and surgical expertise. In the author's experience, superficial masses with $<5$-mm depth of penetration into the renal parenchyma can be excised without renal hilar clamping or suturing. Hilar clamping is recommended, but suturing is still not mandatory, as long as the excision plane does not enter the renal sinus or intra-renal collecting system. For masses that necessitate entry into the renal sinus or intra-renal collecting system, both renal hilar clamping and sutured closure of the renal defect are needed. ${ }^{1}$ Renal hilar dissection and clamping is within the skill set of any urologist capable of performing laparoscopic radical nephrectomy, but the renal parenchymal closure requires more advanced skills, specifically experience in laparoscopic suturing. Although robotic assistance can be of benefit to the laparoscopically naive, ${ }^{2}$ this is still a critical step of the procedure that requires both speed and accuracy. In this article, a method of creating and using the suturing supplies for laparoscopic partial nephrectomy is presented, with the goal of simplifying this challenging step.

\section{Equipment}

Aside from the general supplies needed for laparoscopic renal surgery, the following are needed for creation and use of the suturing supplies for closure of the renal defect:

- Oxidized cellulose fabric, $3 \times 4$ inches (Surgicel Nu-KnitJohnson \& Johnson, New Brunswick, NJ)

- 0-Polyglycolic acid sutures on a CT-1 needle (Ethicon, Somerville, NJ)
- Plastic clips (Hem-o-lok clips; Weck, Raleigh, NC)

- High-quality laparoscopic needle holders

- Eight-inch lengths of 2-0 polyglycolic acid suture on an SH needle (Ethicon)

- Resorbable clips (Lapra-Ty II clips; Ethicon)

- Thrombin/gelatin granules (Floseal; Baxter, Deerfield, IL) with laparoscopic applier

\section{Back-Table Creation of Sutured Bolster}

The Surgicel Nu-Knit oxidized cellulose fabric is available in four sizes: $1 \times 1$ inch, $1 \times 3.5$ inch, $3 \times 4$ inch, and $6 \times 9$ inch. We use the $3 \times 4$-inch piece. Fold the oxidized cellulose fabric in half, and then roll it tightly (Fig. 1). For most defects, folding the fabric length-wise and rolling it perpendicular to the width provides a bolster that is 1.5 inches $(3.8 \mathrm{~cm})$ long and of adequate thickness to provide the necessary volume. One piece of fabric rolled without first folding it in half is too skinny; therefore, for larger resections, place two pieces of the $3 \times 4$-inch oxidized cellulose fabric together and roll perpendicular to the unfolded width, creating a bolster that is 3 inches $(7.6 \mathrm{~cm})$ long. Place a 0 -polyglycolic acid suture on a CT-1 needle through just the side of the bolster, halfway from the ends (Fig. 2), and then secure the suture to the bolster with a knot (Fig. 3), leaving a length of 2 inches from the bolster to the needle. Wrap the free end of the suture around the bolster and knot it securely to hold the bolster rolled tightly (Fig. 4). Cut the suture short (Fig. 5). Use the 0-polyglycolic acid suture left over from the first securing stitch to wrap tightly around one end of the bolster, and then repeat on the other end, such that the bolster is now held in a tight roll by three sutures (Fig. 6). Place a second 0-polyglycolic acid stitch with a CT-1 needle into the bolster, adjacent to the first one (Fig. 7), to complete the bolster (Fig. 8). Both sutures should be placed on the same

Department of Urology, University of Michigan Health System, Ann Arbor, Michigan. 


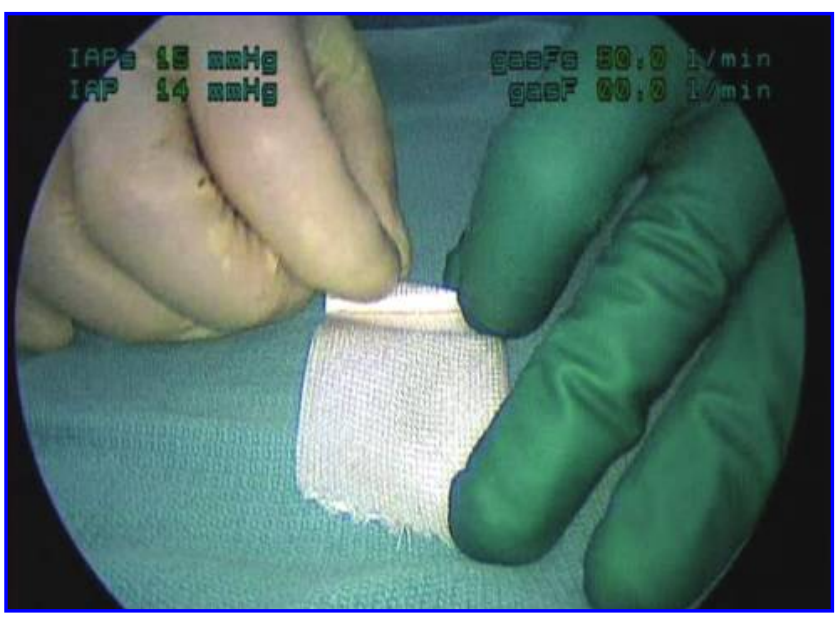

FIG. 1. Fold the oxidized cellulose fabric (Surgicel Nu-Knit) length-wise and roll it along the width.

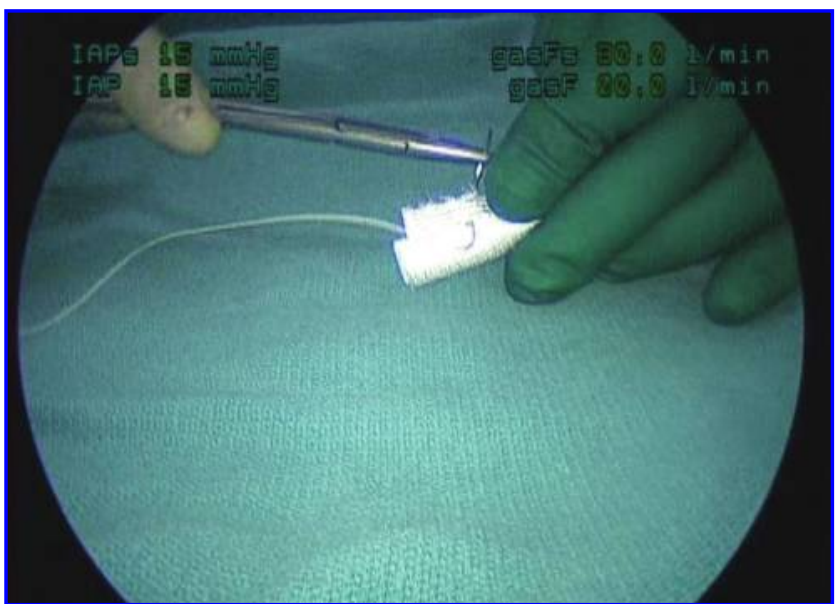

FIG. 2. Place the first 0-polyglycolic acid suture on a CT-1 needle on the side of the bolster, halfway from the ends.

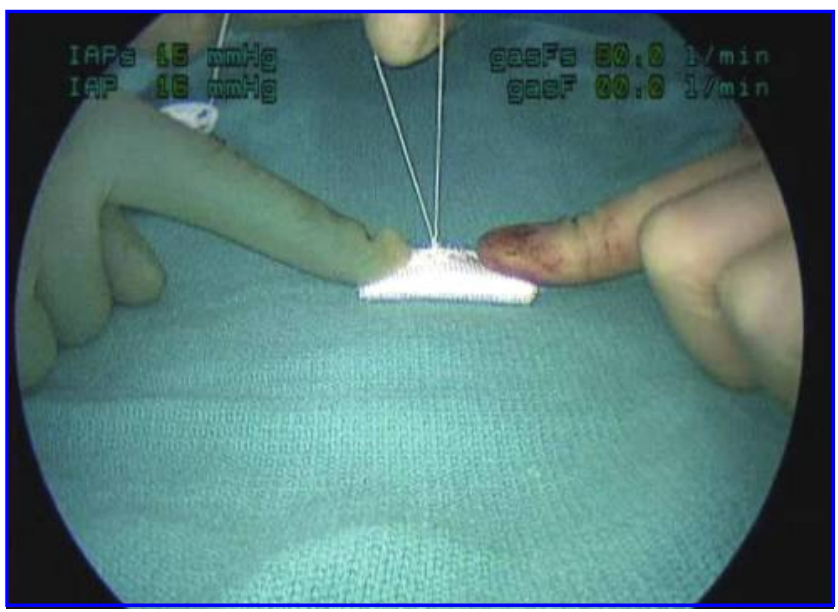

FIG. 3. Secure the suture with a knot.

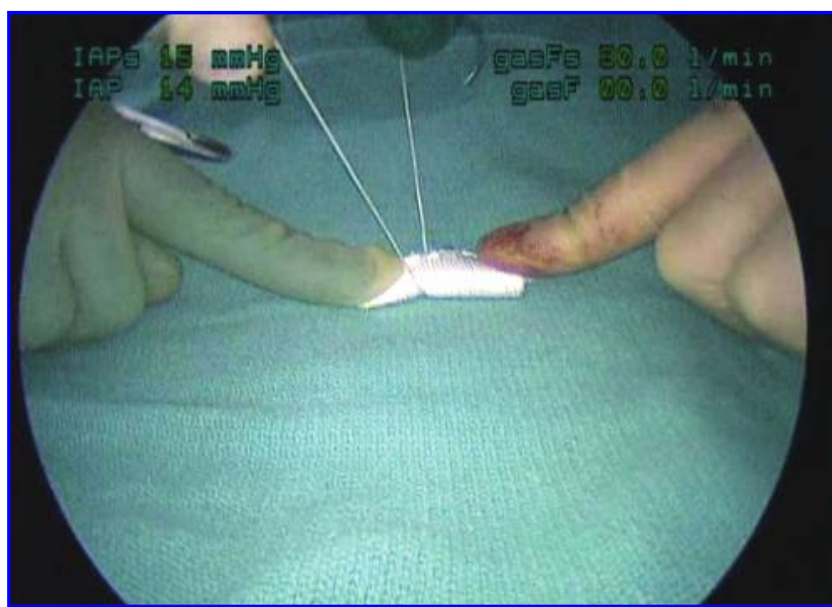

FIG. 4. Wrap the free end of the suture around the bolster to hold it tightly rolled, and knot it securely.

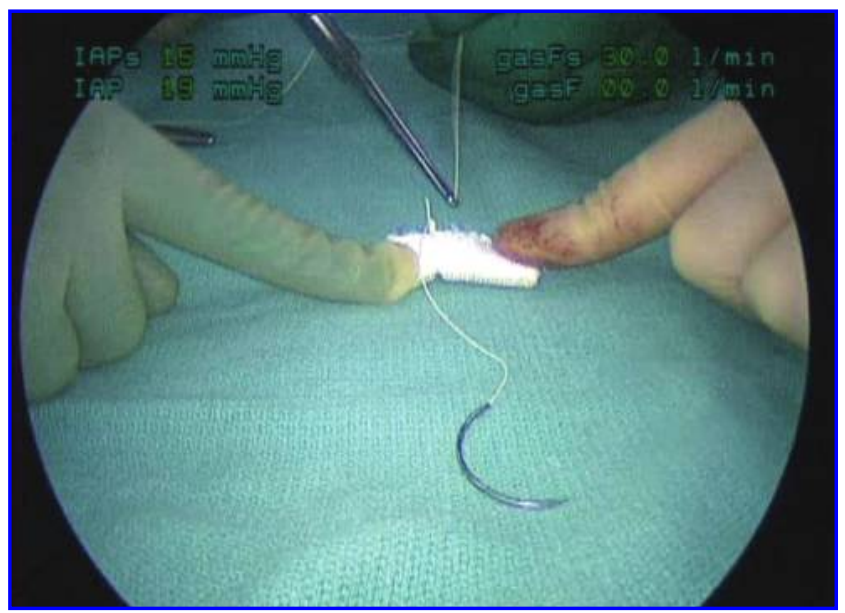

FIG. 5. Cut the suture short.

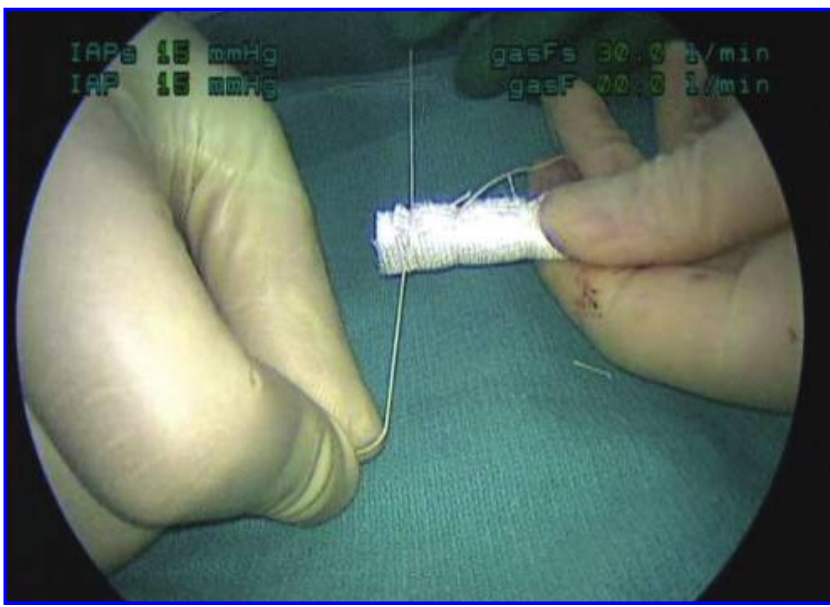

FIG. 6. Use the 0-polyglycolic acid suture left over from first securing stitch to wrap tightly first around one end of the bolster and then the other. 


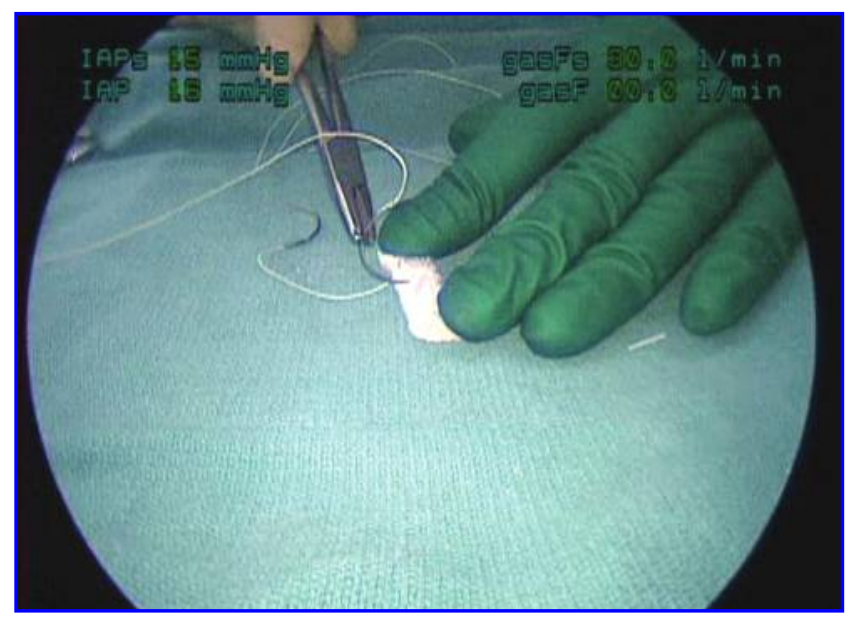

FIG. 7. Place a second 0-polyglycolic acid stitch with a CT-1 needle into the bolster, adjacent to the first one.

side of the bolster, such that there is a top (where both sutures are secured) and a bottom, and both sutures should be in the middle so that the bolster is perpendicular to the sutures when they are pulled taut (Fig. 9).

The sutures that are attached to the bolster are for primary fixation. For secondary fixation, prepare at least two additional sutures. Place a Hem-o-lok clip 3 inches from the CT-1 needle of another 0-polyglycolic acid suture (Fig. 10). Knot the suture on the clip to prevent slippage (Fig. 11). The completed 0-polyglycolic acid suture on CT-1 needle with attached Hemo-lok clip is shown in Figure 12, and Figure 13 illustrates the completed bolster (with attached 0-polyglycolic acid suture and CT-1 needles) and two completed 0-polyglycolic acid sutures on CT-1 needles with attached Hem-o-lok clips. The 8-inch lengths of 2-0 polyglycolic acid suture on an SH needle are not shown.

\section{Sutured Closure of Renal Defect}

Before resection of the renal mass, bring the bolster and the 0-polyglycolic acid sutures on CT-1 needles with attached

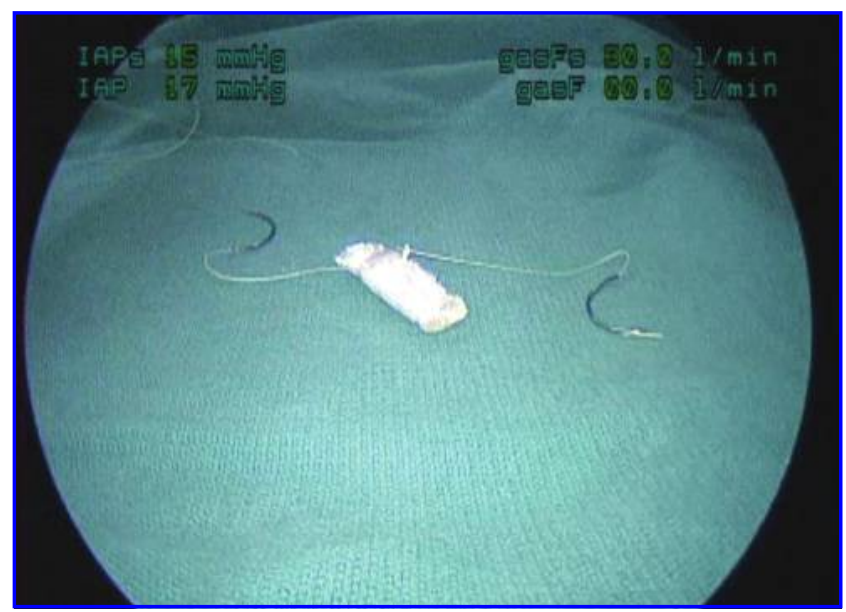

FIG. 8. Completed bolster.

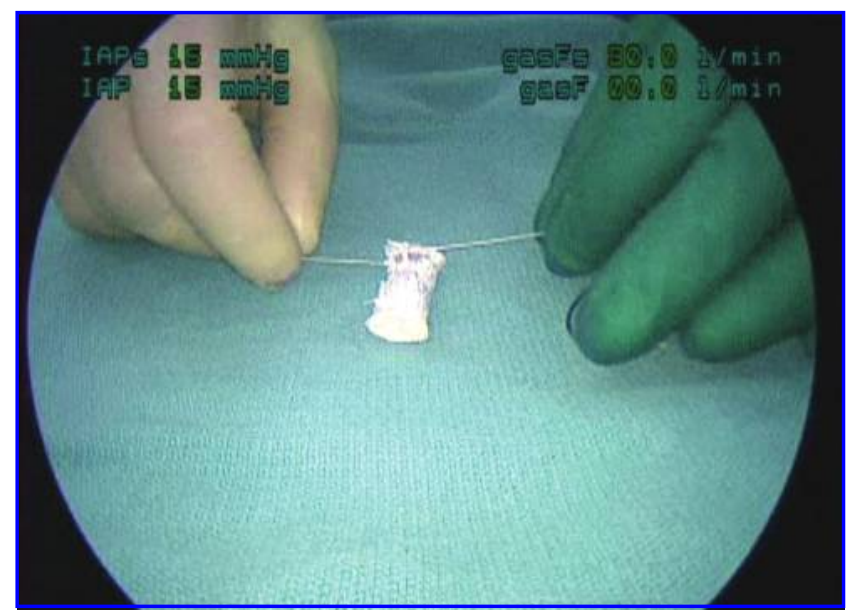

FIG. 9. Pulling the sutures taut confirms that both sutures are halfway from the ends of the bolster, and that there is a top (where both sutures are secured) and a bottom.

Hem-o-lok clips into the abdomen and attach them loosely to the lateral abdominal wall (Fig. 14). Having them in the abdomen before the hilar clamp is applied reduces ischemia time. After resecting the mass and fulgurating the outer rim of parenchyma with an argon beam coagulator, inspect the base of the resection site. If the renal sinus opening is much smaller than the bolster, and if there are no large defects in vessels or intra-renal collecting system, then proceed to bolster placement. If, however, the renal sinus is widely entered, such that the opening into the renal sinus approaches the size of the bolster, or if there are large entries into vessels or the intrarenal collecting system, then use 2-0 polyglycolic acid suture on an $\mathrm{SH}$ needle to place figure-of-eight stitches to close any large defects of vessels or intra-renal collecting system (Fig. 15), and then loosely approximate the parenchymal edges around the sinus opening with a running suture. These knots can be tied free-hand, or a Lapra-ty clip can be used. ${ }^{3}$

Place the bolster with the two attached sutures into the renal defect, aligning it along the long axis of the defect, with

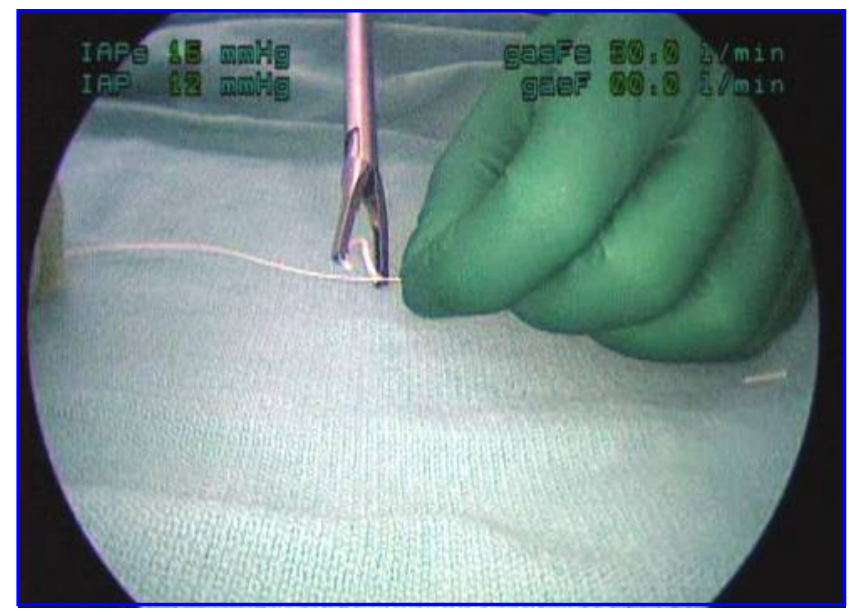

FIG. 10. Place a Hem-o-lok clip 3 inches from the CT-1 needle on the third and fourth 0-polyglycolic acid sutures. 


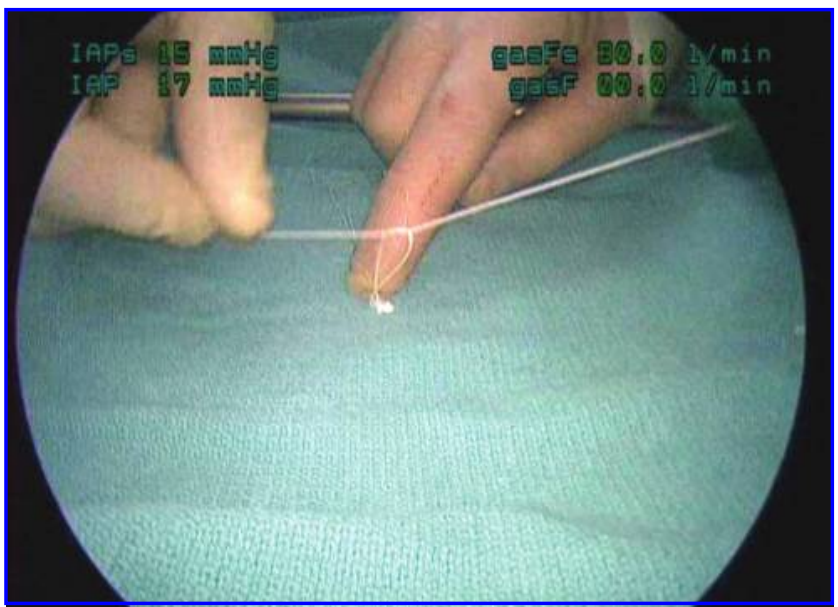

FIG. 11. Knotting the suture on the clip provides additional security.

the top side of the bolster most superficial. Insert each of the attached sutures inside-out into the renal parenchyma halfway between the ends of the defect (Fig. 16). Place a small amount of thrombin/gelatin granules underneath the bolster, and then secure each suture with a Hem-o-lok clip snugly on the parenchymal side (Fig. 17). Complete the bolster fixation by inserting one 0-polyglycolic acid suture on a CT-1 needle with attached Hem-o-lok clip outside-in on the parenchyma at one apex of the resection defect, then over or through the end of the bolster, and finally inside-out on the parenchyma on the other side of the bolster (Fig. 18). Secure this stitch with a Hem-o-lok clip, and then repeat with the other suture at the other apex. In most cases three fixation points are adequate (counting the first two attached sutures as one fixation point), but for large defects use additional 0 -polyglycolic acid sutures with CT-1 needles and Hem-o-lok clips.

When placing the Hem-o-lok clips, it is necessary to only to pull the suture taut and then push the clip snug against the parenchyma. When the vascular clamp is removed the kidney

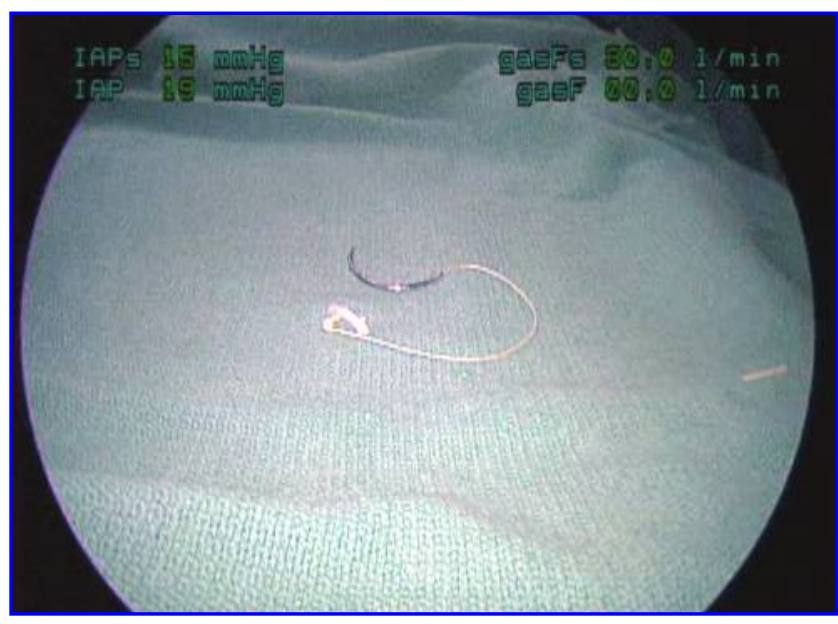

FIG. 12. Completed 0-polyglycolic acid suture on CT-1 needle with attached Hem-o-lok clip.

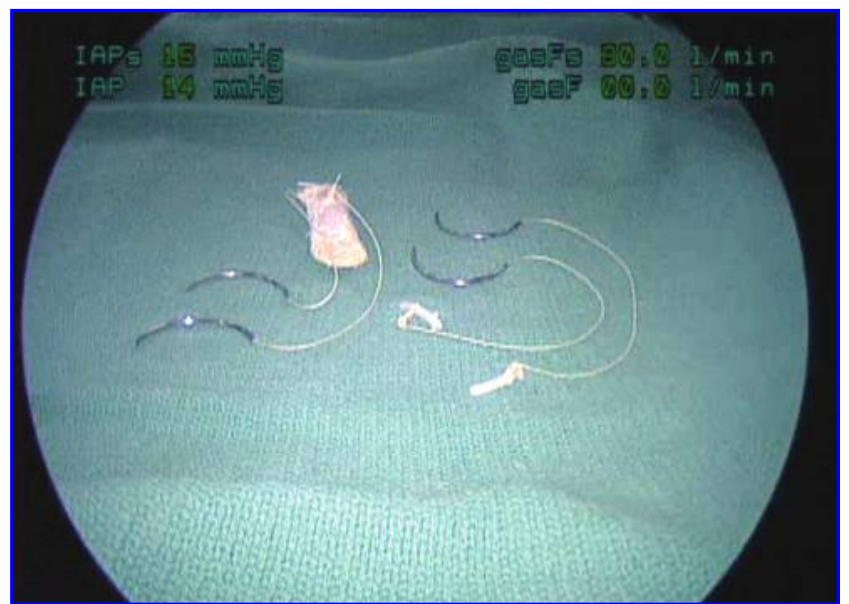

FIG. 13. Completed sutures and bolsters for laparoscopic partial nephrectomy (the 8-inch lengths of 2-0 polyglycolic acid suture on an $\mathrm{SH}$ needle are not shown).

swells and the snug suture tightens down further. This tightening pushes the bolster against the renal parenchyma and provides the compression that is the primary hemostatic mechanism of this technique. There is no need to pull the suture and place the clip with great force; doing so only risks ripping the suture through the parenchyma. When the renal parenchyma is just dimpled by the tension on the clip, then the right amount of force has been applied. If a misjudgment has been made and the clip is not snug, the clip can be pushed down with a grasper or a new clip applied. Leaving a bit of a tail on the suture when cutting the needle off is useful for this maneuver.

Place the remaining aliquot of thrombin/gelatin granules on top of the bolter (Fig. 19), and cover the entire site with the another sheet of oxidized cellulose fabric. This last step allows a surface on which to apply gentle pressure without disturbing the thrombin/gelatin granules.

\section{Discussion}

Although the sutureless laparoscopic partial nephrectomy has been promulgated by some, and the experience in our institution is that $35 \%$ of laparoscopic partial nephrectomies can be performed without suturing, ${ }^{1}$ the author recommends suturing of the renal parenchyma when the resection enters the renal sinus or intra-renal collecting system owing to an unacceptable rate of hemorrhage when suturing is not used in this setting ${ }^{4}$ (at least with current technology). The method of creating and using the suturing material has been developed over several hundred cases at the author's institution. We have found several advantages with this technique. Preattaching the first two sutures to the bolster saves time and prevents slippage of the bolster under the suture. Placing those two sutures halfway from each end of the bolster and on the top firmly seats the bolster in the defect. With this firm fixation, only two additional bolster sutures are needed in most cases.

In summary, the technique of creating and using the suturing supplies presented in this article can be used to simplify the challenging suturing tasks during laparoscopic partial nephrectomy. 


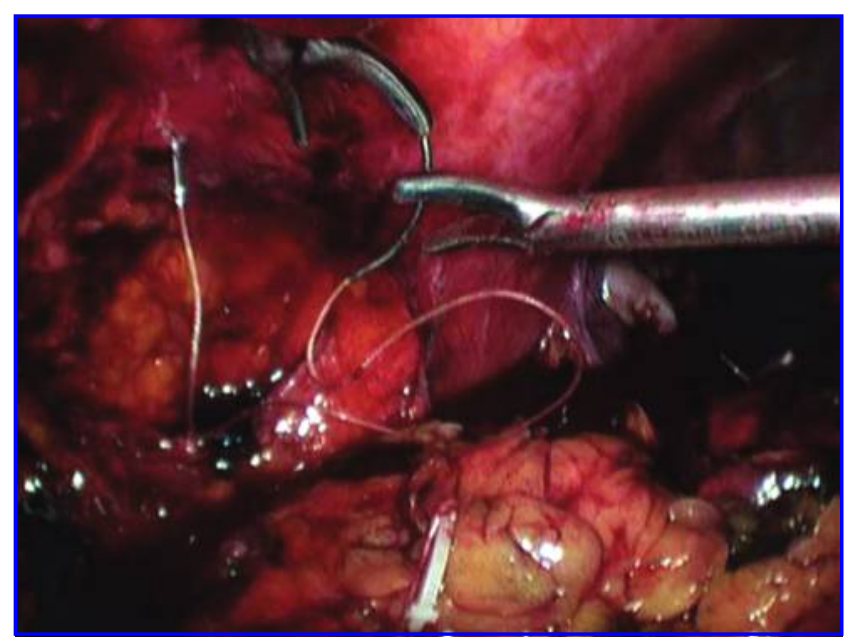

FIG. 14. Secure the bolster and CT-needles loosely to the lateral abdominal wall.

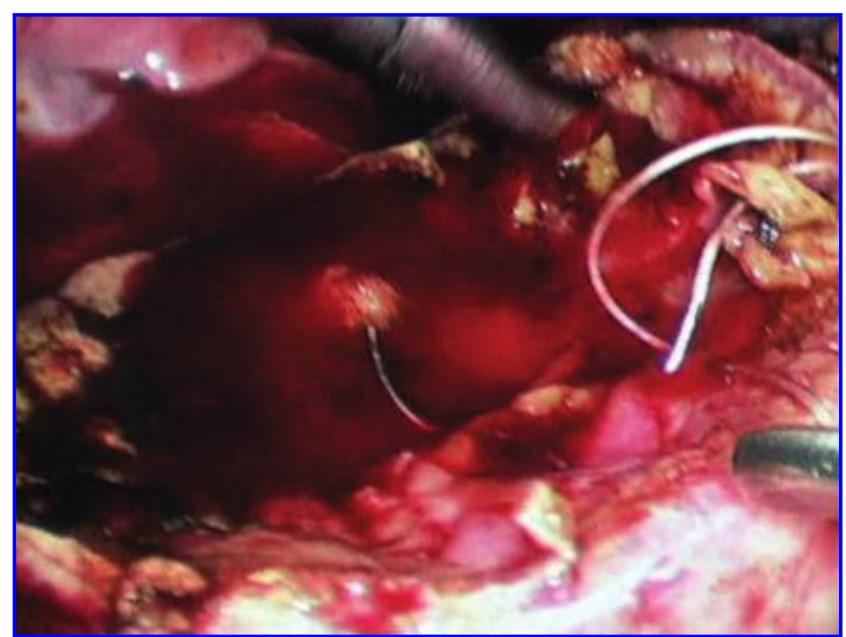

FIG. 15. Use 8-inch lengths of 2-0 polyglycolic acid suture on an $\mathrm{SH}$ needle to approximate the defect in the renal sinus, and to close any large defects in vessels or intra-renal collecting system.

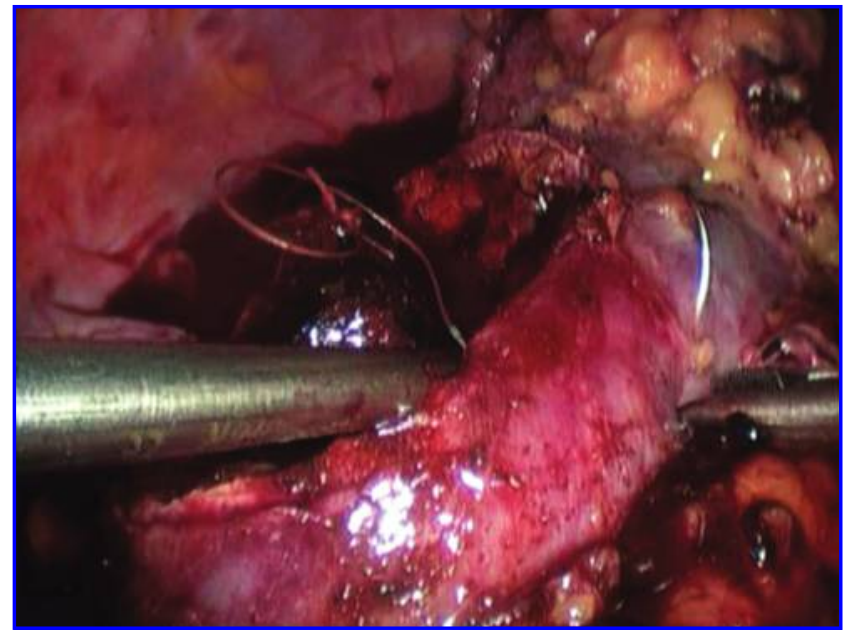

FIG. 16. Place the bolster into the renal defect and insert each of the attached sutures inside-out into the renal parenchyma halfway between the ends of the defect.

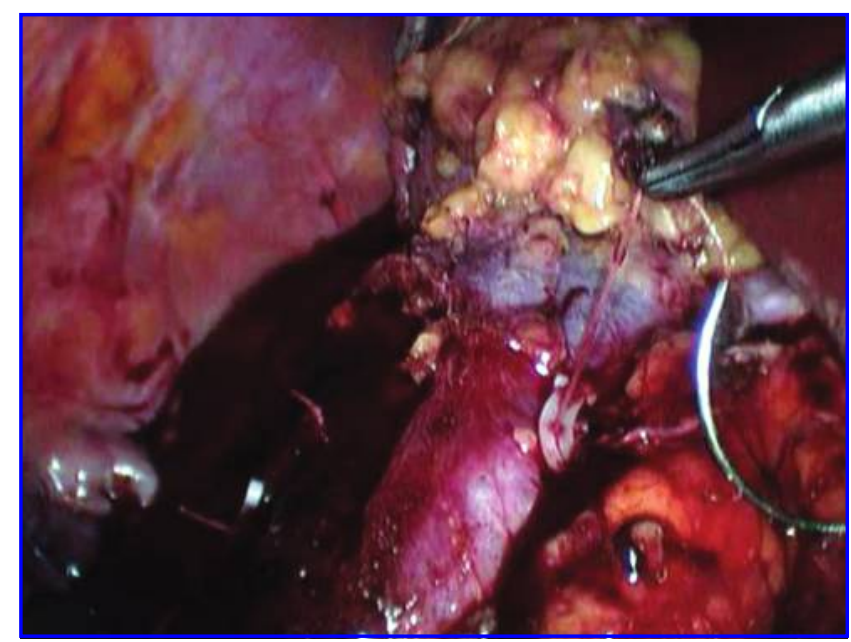

FIG. 17. Clip each end of the suture with a Hem-o-lok clip.

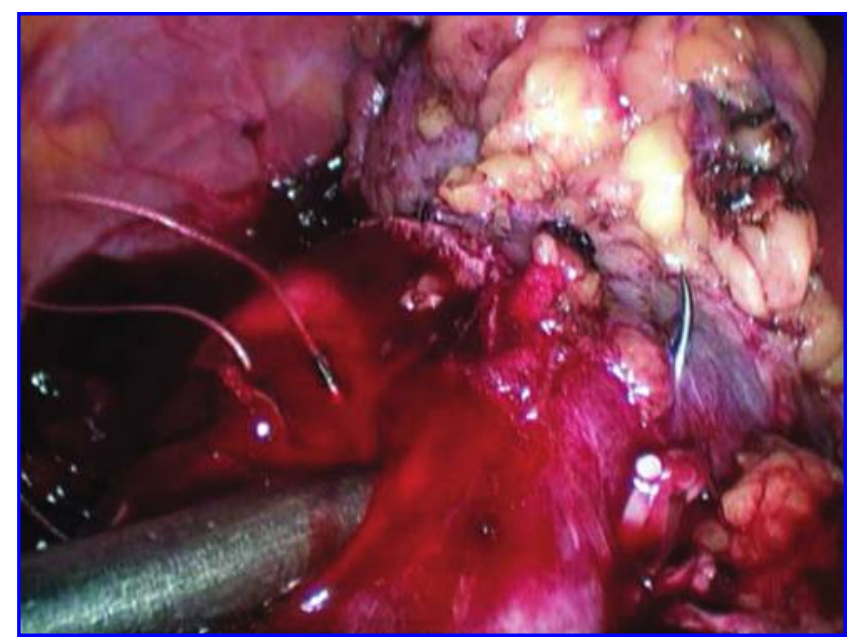

FIG. 18. Place the two additional 0-polyglycolic acid sutures on a CT-1 needle on each end of the defect.

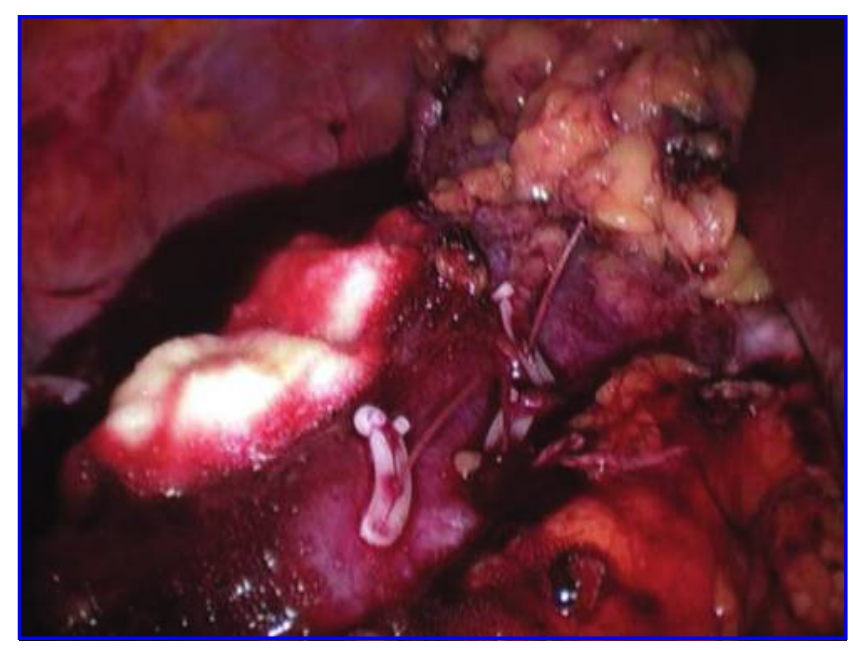

FIG. 19. Completed closure (except for final covering piece of oxidized cellulose fabric). 


\section{References}

1. Weizer AZ, Gilbert SM, Roberts WW, Hollenbeck BK, Wolf JS Jr. Tailoring technique of laparoscopic partial nephrectomy to tumor characteristics. I Urol 2008;180: 1273-1278.

2. Deane LA, Lee HJ, Box GN, et al. Robotic versus standard laparoscopic partial/wedge nephrectomy: A comparison of intraoperative and perioperative results from a single institution. J Endourol 2008;22:947-952.

3. Shalhav AL, Orvieto MA, Chien GW, Mikhail AA, Zagaja GP, Zorn KC. Minimizing knot tying during reconstructive laparoscopic urology. Urology 2006;68:508-513.
4. Johnston WK III, Montgomery JS, Seifman BD, Hollenbeck BK, Wolf JS Jr. Fibrin glue v sutured bolster: Lessons learned during 100 laparoscopic partial nephrectomies. J Urol 2005; 174:47-52.

Address correspondence to: J. Stuart Wolf, Jr., M.D. Department of Urology University of Michigan Health System 1500 East Medical Center Drive, TC 3875 Ann Arbor, MI 48109

E-mail: wolfs@umich.edu 